\title{
THE ASYMPTOTIC BEHAVIOUR OF THE REDUCED MINIMUM MODULUS OF A FREDHOLM OPERATOR
}

\author{
K.-H. FÖRSTER AND M. A. KAASHOEK
}

ABSTRACT. Let $\gamma(S)$ denote the reduced minimum modulus of a linear operator $S$ acting in a complex Banach space $X$, and let $I$ denote the identity on $X$. In this paper it is shown that for a (not necessarily bounded) Fredholm operator $T$ acting in $X$, the $\operatorname{limit}_{n \rightarrow \infty} \gamma\left(T^{n}\right)^{1 / n}$ exists and is equal to the supremum of all positive numbers $\delta$ such that the dimension of the null space and the codimension of the range of $T-\lambda I$ are constant on $0<|\boldsymbol{\lambda}|<\delta$.

Introduction. Throughout this paper $T$ will be a linear operator with domain $D(T)$ and range $R(T)$ in the complex Banach space $X$. By definition the reduced minimum modulus $\gamma(T)$ of $T$ is the supremum of all real numbers $\gamma$ such that

$$
\|T x\| \geq \gamma d(x, N(T)), \quad x \in D(T)
$$

(cf. [6, p. 231] and [3, Definition IV.1.3]). Here $d(x, N(T))$ denotes the distance of $x$ to the null space $N(T)$ of $T$. Observe that we do not require $N(T)$ to be closed in $X$. In [3] $\gamma(T)$ is called the minimum modulus of $T$, but in the present paper this term is reserved for the object studied in [2].

Let $n(T)$ denote the dimension of $N(T)$ and $d(T)$ the codimension of $R(T)$ in $X$. We call $T$ a Fredholm operator if $T$ is a closed linear operator with $n(T)$ and $d(T)$ both finite. If $T$ is a closed linear operator with closed range such that at least one of the numbers $n(T)$ and $d(T)$ is finite, then $T$ is said to be a semi-Fredholm operator. Since the range of a Fredholm operator is closed (cf. [5, Lemma 332]), any Fredholm operator is semi-Fredholm. In $\$ 1$ we show that for a semi-Fredholm operator $T$

$$
\lim _{n \rightarrow \infty} \gamma\left(T^{n}\right)^{1 / n}
$$

exists. Further we compute the limit (1) for a few examples.

Received by the editors January 29, 1974.

AMS (MOS) subject classifications (1970). Primary 47B30, 47A55; Secondary $47 \mathrm{~A} 10$.

Key words and phrases. Fredholm and semi-Fredholm operators, reduced minimum modulus, stability theory, spectrum. 
Suppose that $T$ is a Fredholm operator. From stability theory (cf. $\$$ IV.5 in [6]) we know that there exists a positive number $\delta$ such that $n(T-\lambda I)$ and $d(T-\lambda I)$ are constant on $0<|\lambda|<\delta$. In $\$ 2$ we shall show that the supremum of all $\delta$ with this property is equal to the limit (1). This result is based on a further elaboration of the stability theorems involved and on the existence of holomorphic relative inverses for certain holomorphic operator valued functions (see [1]). As a corollary we obtain a generalization of [2, Theorem 3.5] to the Fredholm set of an operator.

1. The existence of $\lim \gamma\left(T^{n}\right)^{1 / n}$.

Lemma 1. Suppose that $N\left(T^{k}\right) \subset R(T)$ for $k=1,2, \ldots$ Then

$$
\gamma\left(T^{n+m}\right) \geq \gamma\left(T^{n}\right) \gamma\left(T^{m}\right) \quad(n, m=1,2, \cdots) .
$$

Proof. Take $x$ in $D\left(T^{n+m}\right)$. For each $u$ in $N\left(T^{n+m}\right)$, we have

$$
d\left(x, N\left(T^{n+m}\right)\right)=d\left(x-u, N\left(T^{n+m}\right)\right) \leq d\left(x-u, N\left(T^{m}\right)\right) .
$$

By induction it follows from our hypotheses that $T^{m}\left[N\left(T^{n+m}\right)\right]=N\left(T^{n}\right)$. Using this together with (3), we see that

$$
\begin{aligned}
d\left(T^{m} x, N\left(T^{n}\right)\right) & =\inf \left\{\left\|T^{m}(x-u)\right\|: u \in N\left(T^{n+m}\right)\right\} \\
& \geq \gamma\left(T^{m}\right) \inf \left\{d\left(x-u, N\left(T^{m}\right)\right): u \in N\left(T^{n+m}\right)\right\} \\
& \geq \gamma\left(T^{m}\right) d\left(x, N\left(T^{n+m}\right)\right),
\end{aligned}
$$

and hence

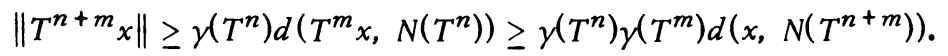

This proves the lemma.

The inequality (2) implies that $\lim \gamma\left(T^{n}\right)^{1 / n}$ exists (see [9, Problem 98]), but not necessarily as a finite number (see Example (1) below). In fact

$$
\rho(T)=\lim _{n \rightarrow \infty} \gamma\left(T^{n}\right)^{1 / n}=\sup _{n \geq 1} \gamma\left(T^{n}\right)^{1 / n} .
$$

In particular, this limit exists if $T$ is injective, and in that case $\rho(T)=$ $\lim _{n \rightarrow \infty} \mu\left(T^{n}\right)^{1 / n}$, where $\mu(T)$ is the minimum modulus as defined in [2].

Theorem 2. If $T$ is a semi-Fredholm operator, then

$$
\rho(T)=\lim _{n \rightarrow \infty} \gamma\left(T^{n}\right)^{1 / n}
$$

exists.

Proof. From our hypotheses it follows that we may apply Theorem 4 in 
[5] to show that $X$ decomposes into two $T$-invariant closed subspaces $X_{0}$ and $X_{1}$ which have the following properties. The space $X_{1}$ is finite dimensional and $X_{1} \subset N\left(T^{k}\right)$ for some $k$. If $T_{0}$ is the restriction of $T$ to $X_{0}$ considered as an operator from $X_{0}$ into itself, then $N\left(T_{0}^{n}\right) \subset R\left(T_{0}\right)$ for $n=1,2, \cdots$. This last fact together with Lemma 1 implies that $\rho\left(T_{0}\right)=\lim \gamma\left(T_{0}^{n}\right)^{1 / n}$ exists. We shall show that $\rho(T)=\rho\left(T_{0}\right)$.

Choose $k$ such that $X_{1} \subset N\left(T^{k}\right)$, and let $P$ be the bounded projection of $X$ along $X_{1}$ onto $X_{0}$. Take $n \geq k$. Since $X_{1} \subset N\left(T^{k}\right) \subset N\left(T^{n}\right) \subset D\left(T^{n}\right)$, it follows that $x \in D\left(T^{n}\right)$ if and only if $P x \in D\left(T_{0}^{n}\right)$, and in that case

$$
T^{n} x=T_{0}^{n} P x
$$

Further, we have for each $x$ in $X$

$$
d\left(x, N\left(T^{n}\right)\right) \leq d\left(P x, N\left(T_{0}^{n}\right)\right) \leq\|P\| d\left(x, N\left(T^{n}\right)\right) .
$$

Together with (4), this implies that

$$
\gamma\left(T_{0}^{n}\right) \leq \gamma\left(T^{n}\right) \leq\|P\| \gamma\left(T_{0}^{n}\right)
$$

and hence

$$
\lim _{n \rightarrow \infty} \gamma\left(T^{n}\right)^{1 / n}=\lim _{n \rightarrow \infty} \gamma\left(T_{0}^{n}\right)^{1 / n}
$$

This proves the theorem.

Examples. (1) Let $X=L_{p}([a, b])$ with $-\infty<a<b<+\infty$ and $1 \leq p$ $\leq \infty$. Let $T$ be the linear operator with domain the set of all absolutely continuous functions $f$ on $[a, b]$ such that $f$ and its derivative $f^{\prime}$ belong to $L_{p}([a, b])$, and suppose that $T f=f^{\prime}, f \in D(T)$. Then $T$ is a surjective linear operator (cf. [3, Theorem VI.3.1]). Thus Lemma 1 implies that $\rho(T)$ exists. By induction one can show that $D\left(T^{n}\right)$ is the set of all $f \in L_{p}([a, b])$ such that the $(n-1)$ th derivative $f^{(n-1)}$ is absolutely continuous on $[a, b]$ and $f^{(n)} \in L_{p}([a, b])$. Further, $T^{n} f=f^{(n)}$ for $f \in D\left(T^{n}\right)$. Hence we can use Example IV.1.4 in [3] to show that $\gamma\left(T^{n}\right) \geq n ! /(b-a)^{n}, n=1,2, \ldots$ Thus $\rho(T)=\infty$.

(2) The inequality (2) does not hold in general; not even if $T$ satisfies the conditions of Theorem 2. To see this take $X=\mathrm{C}^{k}$ with $k>1$ and the Euclidean norm, and define $T: X \rightarrow X$ by $T x=\left(x_{1}, x_{1}, \ldots, x_{1}\right)$, where $x=$ $\left(x_{1}, x_{2}, \ldots, x_{k}\right)$. Then $\|T x\|=\sqrt{k}\left|x_{1}\right|$ and $d(x, N(T))=\left|x_{1}\right|$ for all $x$. Thus $\gamma(T)=\sqrt{k}$. Since $T^{2}=T$, we have $\gamma\left(T^{n}\right)=\sqrt{k}$ for $n=1,2, \ldots$. As $k>1$, this implies that (2) does not hold in this case.

There are other cases in which $\lim \gamma\left(T^{n}\right)^{1 / n}$ exists than those covered by Lemma 1 and Theorem 2. For instance, if $T$ is everywhere defined, 
bounded and nilpotent, then $\gamma\left(T^{n}\right)=+\infty$ for $n$ sufficiently large, and it follows that $\lim \gamma\left(T^{n}\right)^{1 / n}=+\infty$. If $X$ is a Hilbert space and $T$ a selfadjoint linear operator, then $\gamma\left(T^{n}\right)=\gamma(T)^{n}$ for $n=1,2, \ldots$. Hence in that case $\rho(T)=\gamma(T)$.

2. Application to Fredholm operators. Define $k(T)$ to be the dimension of the quotient space

$$
N(T) /\left[\left\{\bigcap_{n=1}^{\infty} R\left(T^{n}\right)\right\} \cap N(T)\right]
$$

(see [4, Definition 2.1]). The condition $k(T)=0$ is equivalent to the requirement that $N\left(T^{n}\right) \subset R(T)$ for $n=1,2, \ldots$. If $T$ is a Fredholm operator, then $k(T)<+\infty$. We need the following special case of Theorem 5.1 in [4].

Theorem 3. If $T$ is a Fredholm operator, then there exists a positive number $\delta$ such that for $0<|\lambda|<\delta$

(a) $T-\lambda I$ is a Fredholm operator with $k(T-\lambda I)=0$,

(b) $n(T-\lambda I)=n(T)-k(T)$,

(c) $d(T-\lambda I)=d(T)-k(T)$.

In the particular case that $k(T)=0$, the constant $\delta$ may be taken to be $\gamma(T)$.

Let $T$ be a Fredholm operator. Define $\delta(T)$ to be the supremum of all $\delta>0$ such that $T-\lambda I$ is a Fredholm operator and $k(T-\lambda I)=0$ for $0<|\lambda|$ $<\delta$. Since $T$ is Fredholm, it follows from Theorem 3 that $\delta(T)$ is the greatest positive number $\delta$ (possibly $\infty$ ) such that $n(T-\lambda I$ ) and $d(T-\lambda I$ ) are constant on $0<|\lambda|<\delta$. The next theorem shows that

$$
\delta(T)=\rho(T)=\lim _{n \rightarrow \infty} \gamma\left(T^{n}\right)^{1 / n} .
$$

In the proof of this theorem we need the following lemma.

Lemma 4. Let $S: X \rightarrow X$ be a bounded linear operator, and suppose that $T S T=T$. Then $\gamma(T) \geq\|S\|^{-1}$.

Proof. Take $x \in D(T)$. Since $S$ is everywhere defined, $x \in D(S T)$. Further, it follows from $T S T=T$ that $T x=T(S T x)$, and thus $x-S T x \in N(T)$. But then

$$
d(x, N(T))=d(S T x, N(T)) \leq\|S T x\| \leq\|S\| \cdot\|T x\| .
$$

This proves the lemma. 
Theorem 5. If $T$ is a Fredholm operator, then

$$
\lim _{n \rightarrow \infty} \gamma\left(T^{n}\right)^{1 / n}
$$

exists and is equal to the supremum of all $\delta>0$ such that $n(T-\lambda I)$ and $d(T-\lambda I)$ are constant on $0<|\lambda|<\delta$.

Proof. From Theorem 2 we know that $\rho(T)=\lim \gamma\left(T^{n}\right)^{1 / n}$ exists. Thus we have to prove the first equality in (6). In order to do this, we first consider the case $k(T)=0$.

Suppose $k(T)=0$. Take $0<|\lambda|<\rho(T)$. Then there exists a positive integer $l$ such that $0<\left|\lambda^{l}\right|<\gamma\left(T^{l}\right)$. Since $T$ is a Fredholm operator, the same is true for $T^{l}$. Further, $k(T)=0$ implies $k\left(T^{l}\right)=0$. Hence we can apply Theorem 3 to show that $T^{l}-\lambda^{l} I$ is a Fredholm operator and $k\left(T^{l}-\lambda^{l} I\right)=0$. So we have

$$
N(T-\lambda I) \subset N\left(T^{l}-\lambda^{l} I\right) \subset R\left(\left(T^{l}-\lambda^{l} I\right)^{n}\right) \subset R\left((T-\lambda I)^{n}\right)
$$

for $n=1,2, \ldots$. But then it follows that $k(T-\lambda I)=0$ and $n(T-\lambda I)$ and $d(T-\lambda I)$ are finite. Since $T$ is closed, the operator $T-\lambda I$ is closed, and thus $T-\lambda I$ is a Fredholm operator with $k(T-\lambda I)=0$. This shows that $\rho(T) \leq \delta(T)$.

To prove the reverse inequality for $k(T)=0$, we have to apply a result on bounded operators. Let $G$ denote the domain $D(T)$ endowed with the graph norm. Then $G$ is a complex Banach space, and, if $\kappa$ is the canonical imbedding of $G$ into $X$, then $\kappa$ and $T \kappa$ are bounded linear operators from $G$ into $X$. Observe that

$$
n(T-\lambda I)=n(T \kappa-\lambda \kappa), \quad d(T-\lambda I)=d(T \kappa-\lambda \kappa) .
$$

In particular, $T-\lambda I$ is Fredholm if and only if $T \kappa-\lambda \kappa$ is Fredholm.

Let $\Delta=\{\lambda \in \mathrm{C}:|\lambda|<\delta(T)\}$. From the definition of $\delta(T)$ and the results of the previous paragraph it follows that $\lambda \mapsto T \kappa-\lambda \kappa$ is a holomorphic Fredholm operator valued function on $\Delta$. Since $k(T)=0$, we have

$$
n(T \kappa-\lambda \kappa)=n(T-\lambda I)=n(T), \quad \lambda \in \Delta .
$$

Hence, by [1, Theorem 5.2], there exists a holomorphic function $B$ on $\Delta$ such that $B(\lambda)$ is a bounded linear operator from $X$ into $G$ and

$$
(T \kappa-\lambda \kappa) B(\lambda)(T \kappa-\lambda \kappa)=T \kappa-\lambda \kappa
$$

for all $\lambda \in \Delta$. Define on $\Delta$ the holomorphic function $Q$ by

$$
Q(\lambda)=I_{G}-B(\lambda)(T \kappa-\lambda \kappa)
$$


where $I_{G}$ denotes the identity operator on $G$. Observe that for each $\lambda$ in $\Delta$, the operator $Q(\lambda)$ is a bounded linear operator on $G$. From (7) it follows that

$$
(T \kappa-\lambda \kappa) Q(\lambda)=0, \quad \lambda \in \Delta .
$$

The holomorphic functions $B$ and $Q$ have power series expansions on $\Delta$.

Let

$$
B(\lambda)=\sum_{n=0}^{\infty} B_{n} \lambda^{n}, \quad Q(\lambda)=\sum_{n=0}^{\infty} Q_{n} \lambda^{n} .
$$

For each $n$, the operator $B_{n}$ is a bounded linear operator from $X$ into $G$ and $Q_{n}$ is a bounded linear operator on $G$. Since the series in (10) converge for all $\lambda$ in $\Delta$, we have

$$
\left\{\lim \sup \left\|B_{n}\right\|^{1 / n}\right\}^{-1} \geq \delta(T) .
$$

From the formulas (8) and (9) it follows that there exist several relations between the coefficients of the power series in (10) and the operator $T$. We shall use these relations to show that the left-hand side of (11) is less than $\rho(T)$.

From (9) we see that

$$
T \kappa Q_{0}=0, \quad T \kappa Q_{n}=\kappa Q_{n-1}, \quad n=1,2, \cdots
$$

By induction this implies that

$$
R\left(\kappa Q_{n}\right) \subset D\left(T^{n+1}\right), \quad T^{n+1} \kappa Q_{n}=0
$$

for $n=0,1,2, \ldots$. From formula (8), we see that

$$
Q_{0}=I_{G}-B_{0} T \kappa, \quad Q_{n}=-B_{n} T \kappa+B_{n-1} \kappa
$$

for $n=1,2, \ldots$. Take a fixed nonnegative integer $k$, and let $x \in D\left(T^{k+1}\right)$. Then $T^{n} x \in D(T)$ for $n=0,1, \cdots, k$. Now $G$ and $D(T)$ considered as sets are equal. Thus $T^{n} x \in G$ and $\kappa\left(T^{n} x\right)=T^{n} x$ for $n=0,1, \cdots, k$. Hence, using (14),

$$
Q_{0} x=x-B_{0} T x
$$

and

$$
Q_{n} T^{n} x=-B_{n} T^{n+1} x+B_{n-1} T^{n} x, \quad n=1, \cdots, k .
$$

We shall show that this implies that

$$
T^{n+1} \kappa B_{n} T^{n+1} x=T^{n+1} x, \quad n=0,1, \cdots, k .
$$


For $n=0$, this follows from (15) and the first part of (12). Proceeding by induction, suppose that $T^{n} \kappa B_{n-1} T^{n} x=T^{n} x$ for some $n$ with $0 \leq n-1<k$. Since $x \in D\left(T^{k+1}\right) \subset D\left(T^{n+1}\right)$, we have $T^{n} x \in D(T)$. Thus $T^{n}\left(\kappa B_{n-1} T^{n} x\right) \epsilon$ $D(T)$, and it follows that $\kappa B_{n-1} T^{n} x \in D\left(T^{n+1}\right)$. Further,

$$
T^{n+1} \kappa B_{n-1} T^{n} x=T^{n+1} x
$$

Also $\kappa Q_{n} T^{n} x \in D\left(T^{n+1}\right)$ by (13). Using this in (16), we see that $\kappa B_{n} T^{n+1} x$ $\in D\left(T^{n+1}\right)$. But then it follows from the second part of (13), (16) and (18) that (17) holds. In particular

$$
T^{k+1} \kappa B_{k} T^{k+1}=T^{k+1}
$$

So we can apply Lemma 4 to show that

$$
\gamma\left(T^{k+1}\right) \geq\left\|\kappa B_{k}\right\|^{-1} \geq\|\kappa\|^{-1}\left\|B_{k}\right\|^{-1}
$$

Since $\|\kappa\| \leq 1$, this implies that

$$
\gamma\left(T^{k+1}\right) \geq\left\|B_{k}\right\|^{-1}, \quad k=0,1,2, \cdots
$$

Using this in (11), it follows that $\rho(T) \geq \delta(T)$. Thus $\rho(T)=\delta(T)$ if $k(T)=0$.

If $k(T) \neq 0$, we apply the reduction process which was used to prove Theorem 2. Let $X_{0}, X_{1}$ and $T_{0}$ be as in the proof of that theorem, and let $I_{0}$ denote the identity operator on $X_{0}$. Since $X_{1}$ is finite dimensional, the operator $\lambda I_{0}-T_{0}$ is a Fredholm operator if and only if $\lambda I-T$ is Fredholm. Hence our hypotheses imply that $T_{0}$ is Fredholm. Further we know that $N\left(T_{0}^{n}\right) \subset R\left(T_{0}\right)$ for $n=1,2, \ldots$. But this implies that $N\left(T_{0}\right) \subset R\left(T_{0}^{n}\right)$ for $n=1,2, \cdots$, and hence $k\left(T_{0}\right)=0$. Therefore, by the result proved above, $\rho\left(T_{0}\right)=\delta\left(T_{0}\right)$. We already know that $\rho(T)=\rho\left(T_{0}\right)$ (see formula (5)). Hence in order to prove the theorem, it remains to show that $\delta(T)=\delta\left(T_{0}\right)$.

Let $T_{1}$ be the restriction of $T$ to $X_{1}$ considered as an operator from $X_{1}$ into $X_{1}$, and let $I_{1}$ denote the identity operator on $X_{1}$. Since $X_{1} \subset$ $N\left(T^{k}\right)$ for some $k$, the operator $T_{1}$ is defined on all of $X_{1}$ and $T_{1}$ is bounded and nilpotent. This implies that $\lambda I_{1}-T_{1}$ is bijective for $\lambda \neq 0$. Hence, for $\lambda \neq 0$,

$$
\begin{gathered}
N(\lambda I-T)=N\left(\lambda I_{0}-T_{0}\right) \\
R\left((\lambda I-T)^{n}\right)=R\left(\left(\lambda I_{0}-T_{0}\right)^{n}\right) \oplus X_{1}, \quad n=1,2, \cdots
\end{gathered}
$$

But then it follows that $k(\lambda I-T)=0$ if and only if $k\left(\lambda I_{0}-T_{0}\right)=0$ for $\lambda \neq 0$. 
We have already observed that $T-\lambda I$ is Fredholm if and only if $T_{0}-\lambda I_{0}$ is Fredholm. Thus $\delta(T)=\delta\left(T_{0}\right)$, and the proof is complete.

By definition the Fredholm set $\Phi(T)$ of $T$ is the set of all complex numbers $\lambda$ such that $T-\lambda I$ is a Fredholm operator. Let

$$
\Delta(T)=\{\lambda \in \Phi(T): k(T-\lambda I)=0\} .
$$

By Theorem 3, the sets $\Phi(T)$ and $\Delta(T)$ are both open in C. If $V$ is a subset of $\mathbf{C}$, we let $d(\lambda, V)$ denote the distance in $\mathbf{C}$ of the point $\lambda$ to $V$. With this notation we have the following corollary to Theorem 5 .

Corollary 6. If $\lambda \in \Phi(T)$, then

$$
\lim _{n \rightarrow \infty} \gamma\left((T-\lambda I)^{n}\right)^{1 / n}=d(\lambda, \mathbf{C} \backslash[\Delta(T) \cup\{\lambda\}]) .
$$

Let $T$ be closed, and suppose that $\lambda$ does not belong to the spectrum $\sigma(T)$ of $T$ (i.e., $T-\lambda I$ is bijective). Then one can use Theorem 3 to show that

$$
d(\lambda, \mathbf{C} \backslash[\Delta(T) \cup\{\lambda\}])=d(\lambda, \sigma(T))
$$

This implies that Theorem 3.5 of [2] is a special case of the above corollary (cf. the remark preceding Theorem 2).

Let $K(X)$ denote the set of compact linear operators on $X$. Define

$$
\gamma_{c}(T)=\sup _{K \in \mathcal{K}(X)} \inf \{\|T x\| /\|x-K x\|: x \in D(T), K x \neq x\} .
$$

Roughly speaking $\gamma_{c}(T)$ is the reduced minimum modulus of $T$ corresponding to the $m$-seminorm introduced by A. Lebow and M. Schechter in [7]. E.-O. Liebetrau [8] has used Theorem 5 of the present paper (for the case that $k(T)=0)$ to show that for $\lambda \in \Phi(T)$

$$
\lim _{n \rightarrow \infty} \gamma_{c}\left((T-\lambda I)^{n}\right)^{1 / n}=d(\lambda, \mathbf{C} \backslash \Phi(T)) .
$$

\section{REFERENCES}

1. H. Bart, Holomorphic relative inverses of operator valued functions, Math. Ann. 208 (1974), 179-194.

2. H. A. Gindler and A. E. Taylor, The minimum modulus of a linear operator and its use in spectral theory, Studia Math. 22 (1962/63), 15-41. MR 27 \#1833.

3. S. Goldberg, Unbounded linear operators: Theory and applications, McGrawHill, New York, 1966. MR 34 \#580.

4. M. A. Kaashoek, Stability theorems for closed linear operators, Nederl. Akad. Wetensch. Proc. Ser. A 68 = Indag. Math. 27 (1965), 452-466. MR 31 \#6129.

5. T. Kato, Perturbation theory for nullity, deficiency and other quantities of linear operators, J. Analyse Math. 6 (1958), 261-322.

6. - Perturbation theory for linear operators, Die Grundlehren der math. Wissenschaften, Band 132, Springer-Verlag, New York, 1966. MR 34 \#3324. 
7. A. Lebow and M. Schechter, Semigroups of operators and measures of noncompactness, J. Functional Analysis 7 (1971), 1-26. MR 42 \#8301.

8. E.-O. Liebetrau, Über die -Fredholmmenge linearer Operatoren, Dissertation, Dortmund, 1972.

9. G. Pólya and G. Szegö, Problems and theorems in analysis. Vol. 1, Die Grundlehren der math. Wissenschaften, Band 193, Springer-Verlag, Berlin and New York, 1972.

FACHBERICH MATHEMATIK, UNIVERSITÄT OLDENBURG, OLDENBURG, GERMANY (Current address of K.-H. Förster)

WISKUNDIG SEMINARIUM, VRIJE UNIVERSITEIT, AMSTERDAM, THE NETHERLANDS

Current address (M. A. Kaashoek): Department of Mathematics, University of Maryland, College Park, Maryland 20742

PROCEEDINGS OF THE

AMERICAN MATHEMATICAL SOCIETY

Volume 49. Number 1, May 1975

\title{
CHARACTERIZATIONS OF THE COMPLEX PROJECTIVE PLANE BY CURVATURE
}

\author{
ROBERT E. GREENE AND HARSH PITTIE
}

ABSTRACT. We give short proofs to show that under various positivity assumptions on the curvature of a Kähler surface $X$, it is biholomorphically equivalent to $P_{2}(C)$. In particular, the case of $\delta$-holomorphic pinching $>1 / 2$ (Theorem 1 ) is best possible and, we believe, new.

Let $X$ be a compact Kähler manifold of complex dimension 2. Given a real two-dimensional subspace $p$ of the real tangent space of $X$ at some point, we denote by $K(p)$ the Riemannian curvature of $p . X$ is called $\delta$-bomomorpbically pinched if there is some constant $A>0$ such that $\delta A \leq K(p) \leq A$ for all holomorphic planes $p$, i.e. for all planes $p$ invariant under the complex structure endomorphism $J$. Given two holomorphic planes

Received by the editors November 13, 1973 and, in revised form, February 11, 1974.

AMS (MOS) subject clas sifications (1970). Primary 32C10, 32J15, 53C20.

Key words and phrases. Kähler manifold, curvature, pinching, Chern class, Pontrjagin class, Riemann-Roch, Kodaira vanishing the orem. 